\title{
Erratum to: Hausdorffness for Lie algebra homology of Schwartz spaces and applications to the comparison conjecture
}

\author{
Avraham Aizenbud $^{1}$. Dmitry Gourevitch ${ }^{1}$. \\ Bernhard Krötz ${ }^{2}$. Gang Liu ${ }^{3}$
}

Published online: 1 June 2016

(C) Springer-Verlag Berlin Heidelberg 2016

\section{Erratum to: Math. Z. DOI 10.1007/s00209-016-1629-6}

The following changes to the main results of [1] are necessary:

(1) In Theorem A and Corollary B the following assumption is required: the number of orbits of the complexification $H_{\mathbb{C}}$ on $G_{\mathbb{C}} / P_{\mathbb{C}}$ is finite, where $P$ is a minimal parabolic subgroup of $G$.

(2) In Theorem $C$ the following additional assumption is required: the number of orbits of $H_{\mathbb{C}}$ on $X_{\mathbb{C}}$ is finite.

Presently we do not know whether these results hold without the additional assumptions.

The source of the mistake is in [2], where the expressions "real algebraic groups" and "real algebraic manifolds" are ambiguous. Moreover, a mistake in [2, Definition 1.1.1] hints to a wrong resolution of this ambiguity, in particular in [2, Theorem D]. This result entered in the proof of Lemma 3.2.1 of [1].

The online version of the original article can be found under doi:10.1007/s00209-016-1629-6.

$\bowtie$ Dmitry Gourevitch

dmitry.gourevitch@weizmann.ac.il

Avraham Aizenbud

aizenr@gmail.com

Bernhard Krötz

bkroetz@gmx.de

Gang Liu

gang.liu@univ-lorraine.fr

1 Faculty of Mathematics and Computer Science, Weizmann Institute of Science, POB 26, 76100 Rehovot, Israel

2 Institut für Mathematik, Universität Paderborn, Warburger Str. 100, 33098 Paderborn, Germany

3 Institut Élie Cartan de Lorraine, Université de Lorraine, Ile du Saulcy, 57045 Metz, France 
In [2] the terms "real algebraic groups" and "real algebraic manifolds" sometimes mean algebraic groups and manifolds defined over $\mathbb{R}$, and sometimes real points of such. Those two meanings are not equivalent; in particular, the statement that an algebraic group $G$ defined over $\mathbb{R}$ acts on an algebraic manifold $X$ with finitely many orbits implies the statement that $G(\mathbb{R})$ acts on $X(\mathbb{R})$ with finitely many orbits, but is not equivalent to it. Rather, it is equivalent to the stronger statement that $G(\mathbb{C})$ has finitely many orbits on $X(\mathbb{C})$. In particular, in [2, Theorem D] one needs the stronger assumption (only then it follows from the BernsteinKashiwara theorem, [2, Thm 3.2.2]), see [3]. We do not know whether this theorem holds under the weaker assumption.

The argument in [1] proves the corrected versions of the main results (see $(1,2)$ above), after the following revision.

(a) In Sects. 2 and 3, the expression "real algebraic group" has to be replaced by "algebraic group defined over $\mathbb{R}$ " and the expression "real algebraic manifold" has to be replaced by "algebraic manifold defined over $\mathbb{R}$ ".

(b) One has to introduce the following notation: for an algebraic manifold $X$ defined over $\mathbb{R}$, a Zariski closed algebraic submanifold $Z$ and an algebraic bundle $\mathcal{E}$ over $X$ denote $\mathcal{S}_{Z}(X, \mathcal{E}):=\mathcal{S}_{Z(\mathbb{R})}(X(\mathbb{R}), \mathcal{E})$ and $\mathcal{S}_{Z}^{*}(X, \mathcal{E}):=\mathcal{S}_{Z(\mathbb{R})}^{*}(X(\mathbb{R}), \mathcal{E})$, and similarly for the special cases $\mathcal{S}(X), \mathcal{S}(X, \mathcal{E}), \mathcal{S}_{Z}(X)$, and their dual spaces.

(c) In the proof of Lemma 3.2.1, one has to add that the reason that Proposition 3.1.1 implies the finiteness of the dimension of

$$
\mathrm{H}_{0}\left(\mathfrak{h}, \mathcal{S}_{Z}(X, \mathcal{E}) / \mathcal{S}_{Z}(X, \mathcal{E})^{i} \otimes \chi\right)
$$

is that $Z(\mathbb{R})$ is a finite union of $H(\mathbb{R})$-orbits.

(d) In Sect. 4, $G$ should be the group of real points of an algebraic reductive group $\mathbf{G}$ defined over $\mathbb{R}$, and $H$ should be the group of real points of an algebraic subgroup $\mathbf{H} \subset \mathbf{G}$. Also, each time that we require $H$ to be a real spherical subgroup we actually need to require the stronger condition that $\mathbf{H}$ has finitely many orbits on $\mathbf{G} / \mathbf{P}$, where $\mathbf{P}$ is a minimal parabolic subgroup of $\mathbf{G}$ defined over $\mathbb{R}$.

\section{References}

1. Aizenbud, A., Gourevitch, D., Kroetz, B., Liu, G.: Hausdorffness for Lie algebra homology of Schwartz spaces and applications to the comparison conjecture. Math. Z. (2016). doi:10.1007/s00209-016-1629-6

2. Aizenbud, A., Gourevitch, D., Minchenko, A.: Holonomicity of relative characters and applications to multiplicity bounds for spherical pairs (old version). arXiv:1501.01479v1

3. Aizenbud, A., Gourevitch, D., Minchenko, A.: Holonomicity of relative characters and applications to multiplicity bounds for spherical pairs. arXiv:1501.01479v2 